\title{
Effect of Modified Sijunzi Decoction on Macrophage Polarization Into M1 Phenotype in a Balb/c Mouse Model of Breast Cancer
}

\author{
huamiao zhou
}

Zhejiang provincial hospital of TCM

\section{Binyue Xu}

Zhejiang Chinese Medical University

\section{Yong Guo}

Zhejiang provincial hospital of TCM

\section{Xiangyun Zhang}

Zhejiang Chinese Medical University

Zhendong Liu ( $\square$ liudong761@163.com )

Zhejiang provincial hospital of TCM https://orcid.org/0000-0002-8339-4054

\section{Research}

Keywords: Modified Sijunzi decoction, breast cancer, tumor growth, metastasis

Posted Date: April 12th, 2021

DOl: https://doi.org/10.21203/rs.3.rs-372785/v1

License: (9) This work is licensed under a Creative Commons Attribution 4.0 International License. Read Full License 


\section{Abstract}

Background: The five-year survival rate of breast cancer is bleak because of the predilection for bone metastasis. Tumor-associated macrophages are involved in tumor metastasis and are divided into two antagonistic types, M1 and M2. This study aimed to detect the anti-tumor effect of the modified Sijunzi decoction (MSJZD) in a mouse model of breast cancer and explore whether MSJZD inhibited tumor metastasis by regulating macrophage polarization.

Materials and methods: A luciferase-expressing mouse breast cancer cell line Luc-4T1 was inoculated into the right mammary fat pad of mice to establish a Balb/c mouse model of breast cancer. After inoculation for $24 \mathrm{~h}$, the mice were randomly divided into the MSJZD group and control group $(\mathrm{n}=5$ per group). The mice in the MSJZD group were gavaged with $0.77 \mathrm{~g} / \mathrm{mL}$ MSJZD once daily for 35 days, whereas those in the control group were administered the same volume of normal saline. Subsequently, the effects of MSJZD on tumor growth and macrophage polarization were investigated.

Results: On day 35, MSJZD reduced tumor growth in the mouse model of breast cancer. Flow cytometry showed that the M1 marker (inducible nitric oxide synthase ${ }^{+}$) was increased in the MSJZD group relative to that in the control group, whereas the M2 marker (CD206 ${ }^{+}$) did not exhibit significant differences between the two groups. The results indicated that MSJZD promoted macrophage polarization into the M1 phenotype.

Conclusions: Our findings showed that MSJZD promoted macrophage polarization into the M1 phenotype, thus inhibiting tumor growth and metastasis in breast cancer.

\section{Introduction}

Breast cancer is one of the most common malignancies in women worldwide. In 2015, it was estimated that approximately 231,840 newly diagnosed invasive cases and 40,290 deaths occurred in the United States (1). Most breast cancer patients are diagnosed at an advanced stage, and the predilection for bone metastasis greatly affects the five-year survival rate $(<10 \%)(2,3)$. Therefore, it is imperative to explore effective treatments to prevent the progression of breast cancer.

Tumor-associated macrophages (TAMs) are a major component of leukocytic infiltrate of tumors and play important role in cancer-related inflammation $(4,5)$. According to the activation type of macrophages, TAMs can be divided into two antagonistic phenotypes: pro-immune (M1) and pro-immune (M1) phenotypes $(6,7)$. M1 macrophages aggressively destroy tumor cells and impact anti-tumor effects by releasing pro-inflammatory cytokines, whereas $\mathrm{M} 2$ macrophages promote tumor growth and metastasis by regulating immune inhibitory signaling molecules $(8,9)$. It is reported that TAMs can recruit to the tumor site and thus promote tumor angiogenesis and metastasis, especially being polarized into M2 phenotype by the tumor microenvironment (10). Moreover, TAMs provide an ideal therapeutic target for suppressing tumor progression after being polarized to a M1 phenotype (11-13). Reversing the 
polarization of TAMs from M2 to M1 phenotypes may be a potential therapeutic strategy for human cancers.

Traditional Chinese medicine (TCM) has been popularly used for the prevention and treatment of various cancers over a long period of time (14-16). It is widely applied as an adjuvant therapy alongside main treatments, such as chemotherapy and radiotherapy, in the treatment of advanced cancers, and has been shown to reduce the toxicity and side effects of chemotherapy $(17,18)$. Because of its anti-tumor effects, a growing body of TCM has gained international recognition and acceptance (19). TCM is also increasingly employed as an adjunctive therapy for breast cancer $(20,21)$. Further identification of the effect of TCM on breast cancer may provide novel strategies for tumor therapy. Sijunzi decoction is a basic prescription for strengthening the spleen and replenishing qi (22) that has been reported to exhibit anti-tumor activity (23). Sijunzi decoction-treated rat serum has been shown to have growth inhibitory effects on side population cells by inducing cell apoptosis in gastric carcinoma (23). However, there is no report on the use of Sijunzi decoction in the treatment of breast cancer and the underlying regulatory mechanisms involved therein. Whether Sijunzi decoction prevents breast cancer development via regulating macrophage polarization into M1 phenotype is largely unknown.

In this study, we aimed to explore the effects of modified Sijunzi decoction (MSJZD) in the treatment of breast cancer. Luciferase-expressing Luc-4T1 mouse breast cancer cells were inoculated into the right mammary fat pad of female Balb/c mice to establish a mouse model of breast cancer. Subsequently, the effects of MSJZD on tumor growth and macrophage polarization in the mouse model of breast cancer were investigated. We found that MSJZD played critical roles in the treatment of breast cancer and might provide new strategies for disease treatment.

\section{Materials And Methods}

\section{Preparation of MSJZD}

MSJZD is composed of ginseng ( $15 \mathrm{~g}$; the place of origin is Gansu, China; purchased from Hangzhou huadong traditional Chinese medicine yinpian co., Ltd., Zhejiang, China), atractylodes (12 g; the place of origin is Zhejiang, China; purchased from Zhejiang qianwang traditional Chinese medicine co. Ltd., Zhejiang, China), Poria cocos ( $15 \mathrm{~g}$; the place of origin is Anhui, China; purchased from Zhejiang zoli baicao decoction piece co. Ltd., Zhejiang, China), honey-fried licorice root (6 g; the place of origin is Xinjiang, China; purchased from Hangzhou huadong traditional Chinese medicine yinpian co., Ltd., Zhejiang, China), zedoary (12 g; the place of origin is Guangxi, China; purchased from Quzhou nankong traditional Chinese medicine co., Ltd., Zhejiang, China), and fruit of five-leaf akebia ( $15 \mathrm{~g}$; the place of origin is Hubei, China; purchased from Zhejiang yingte traditional Chinese medicine yinpian co. Ltd., Zhejiang, China). These herbs were immersed in distilled water for $2 \mathrm{~h}$, decocted, filtered, condensed to $0.77 \mathrm{~g} / \mathrm{mL}$ with a routine method, and kept at $4^{\circ} \mathrm{C}$ for use.

\section{Establishment of the mouse model of breast cancer}


Ten 8-week-old female Balb/c mice weighing $20 \pm 2 \mathrm{~g}$ (purchased from the Laboratory Animal Center of Zhejiang Chinese Medical University) were maintained in a specific-pathogen-free room at constant temperature $\left(24^{\circ} \mathrm{C} \pm 2^{\circ} \mathrm{C}\right)$ and humidity $(55 \% \pm 5 \%)$ under a $12 / 12$-h light/dark cycle. Luciferaseexpressing Luc-4T1 breast cancer cells derived from the epithelium of female Balb/c mice (Caliper Life Sciences, Hopkinton, MA) were inoculated into the right mammary fat pad of anesthetized mice at a dose of $5 \times 10^{6}$ cells $/ \mathrm{mL}$ in $0.1 \mathrm{~mL}$ of normal saline per animal. After inoculation for $24 \mathrm{~h}$, the mice were randomly divided into the MSJZD group and control group ( $n=5$ per group). The mice in the MSJZD group were gavaged with $0.77 \mathrm{~g} / \mathrm{mL}$ MSJZD once daily for 35 days. The mice in the control group were administered the same volume of normal saline. On day 35 , the mice were sacrificed by cervical dislocation, and the tumors were harvested and weighed. The animal experiments were conducted with the approval of the Institutional Animal Care and Use Committee of Zhejiang Chinese Medical University.

\section{In vivo image analysis}

To detect tumor growth and metastasis, in vivo imaging study was performed on days $15,22,29,32$, and 35. The mice were intraperitoneally injected with $1.5 \mathrm{mg} / 10 \mathrm{~g}$ body weight of fluorescent DiD oil. Six hours after the injection, the mice were anesthetized via isoflurane inhalation and imaged using a Caliper Life Sciences in vivo imaging system (MA, USA). By means of living imaging software (version 4.3.1, Xenogen, Alameda, USA), image display analysis was conducted and data were collected by drawing regions of interest on the subcutaneous tumors. By calculating the number of photons emitted from the tumor site of the mice, the fluorescence intensity (radiance) was measured.

\section{Macrophage quantification by flow cytometry}

After 35 days, TAMs were analyzed by flow cytometry based on the amount of M1/M2 macrophages. For cell sorting, the mouse tumor cells were harvested by trypsinization with $0.25 \%$ trypsinethylenediaminetetraacetic acid (Invitrogen; Thermo Fisher Scientific, Inc.) and then re-suspended in prewarmed $\left(37^{\circ} \mathrm{C}\right)$ Dulbecco's modified Eagle's medium (Gibco; Thermo Fisher Scientific, Waltham, MA, USA) containing $2 \%$ fetal bovine serum. The density of the cells was adjusted to $1 \times 10^{6}$ cells $/ \mathrm{mL}$ and the cells were passed through 40- $\mu \mathrm{m}$ cell strainers (BD Falcon; BD Biosciences, San Jose, CA, USA) to obtain single cell suspensions. To characterize the infiltration of M1 and M2 macrophages, the cells were stained for $20 \mathrm{~min}$ at room temperature with the following antibodies: phycoerythrin anti-mouse F4/80 (BioLegend, San Diego, CA), rabbit anti-human inducible nitric oxide synthase (iNOS, eBioscience, San Diego, CA, USA), and mouse anti-human CD206 (BioLegend). The cells were then subjected to flow cytometry on a BD FACSCantoll apparatus (BD Biosciences) and the data were analyzed using the CellQuest software.

\section{Statistical analysis}

The measurement data obtained from at least three independent assays in each experiment were expressed as the mean \pm standard deviation. Statistical differences between two groups were compared through one-way analysis of variance. All statistical analyses were conducted using the SPSS Statistics 17.0 software (IBM, Armonk, NY, USA). Statistical significance was indicated when $\mathrm{P}<0.05$. 


\section{Results}

\section{MSJZD reduced tumor growth in the mouse model of breast cancer}

On day 35, the tumors were harvested from the control and MSJZD-treated mice and the tumor weights were $952.465 \pm 316.657$ and $785.091 \pm 171.853 \mathrm{mg}$, respectively. The tumor weight and size of the MSJZD-treated mice were slightly lower than those of the control mice, but there were no significant differences (Fig. 1A). In vivo imaging performed on the tumor-induced mice on days 15, 22, 29, 32, and 35 revealed that the numbers of photons emitted from the tumor site of the MSJZD-treated mice were $5.54 \pm$ $5.84,9.44 \pm 5.07,6.43 \pm 4.41,17.45 \pm 3.23$, and $1.54 \pm 0.31\left(\times 10^{6}\right.$, photons $\left./ \mathrm{s} / \mathrm{cm}^{2} / \mathrm{sr}\right)$, respectively; and those in the control group were $2.22 \pm 1.43,2.92 \pm 1.94,2.78 \pm 1.93,3.68 \pm 1.65$, and $1.55 \pm 0.29\left(\times 10^{6}\right.$, photons $/ \mathrm{s} / \mathrm{cm}^{2} / \mathrm{sr}$ ), respectively (Fig. 1B).

\section{MSJZD promoted macrophage polarization into M1 phenotype}

The effect of MSJZD on macrophage polarization was explored to elucidate the anti-tumor role of MSJZD. The proportions of the M1 marker $\left(\mathrm{iNOS}^{+}\right)$and $\mathrm{M} 2$ marker $\left(\mathrm{CD} 206^{+}\right)$were detected by flow cytometry. We found that the expression of the $\mathrm{M} 1$ marker $\left(\mathrm{iNOS}^{+}\right.$) was significantly increased in the MSJZD group compared with that in the control group $(P<0.01)$, while the $M 2$ marker $\left(C D 206^{+}\right)$did not exhibit significant differences between the two groups $(P>0.05)$ (Fig. 2). These results suggested that MSJZD exerted anti-tumor effects by promoting macrophage polarization into the M1 phenotype.

\section{Discussion}

Malignant breast cancer is a major health issue among women worldwide. Earlier detection combined with effective treatment may reduce the mortality of breast cancer. Sijunzi decoction is a well-known TCM formulation that exerts potential effects on immune system activation (24) and exhibits anti-tumor activity (23). In this study, MSJZD was used to treat breast cancer in an animal model and its effect on tumor growth and macrophage polarization was evaluated. The results showed that MSJZD reduced tumor growth in a Balb/c mouse model of breast cancer and promoted macrophage polarization into the M1 phenotype. These findings merit further discussion.

Sijunzi decoction has been shown to exhibit numerous pharmacological effects, such as regulating gastrointestinal motility, stimulating digestion and absorption, and promoting intestinal mucosal immunity $(24,25)$. Moreover, previous studies have confirmed that Sijunzi decoction controlled tumor progression, prolonged patient survival (26), and prevented tumor recurrence and metastasis $(27,28)$. Increasing studies have confirmed the anti-tumor activity of Sijunzi decoction in animal models and cancer cell lines. For instance, supplementary Sijunzi decoction was shown to remarkably prevent N- 
methyl-N'-nitro-N-nitrosoguanidine-induced gastric cancer in a rat model (29). The ingredients of Sijunzi decoction were found to decrease the proliferation of the side population of BGC-823 cells by inducing G1/G0 phase arrest and apoptosis (30). It is was also reported that MSJZD exhibited immune-enhancing effects on colorectal cancer patients undergoing chemotherapy (31). Consistently, our results revealed that MSJZD suppressed tumor growth in a Balb/c mouse model of breast cancer, suggesting the antitumor effect of MSJZD in breast cancer.

Increasing evidence has indicated that TAMs play important roles in tumor microenvironment formation, tumor metastasis, and anti-tumor responses (32-35). A previous study demonstrated that TAMs are related to the aggressive features of breast cancer, such as vascular invasion and reduced survival (36). The polarization of TAMs from M1 to M2 phenotypes is a hallmark of malignancy (37). A high M1/M2 ratio in TAMs has been reportedly associated with extended survival in ovarian cancer patients (38). In addition, Kang et al. revealed that puerarin stimulated anti-tumor effects in non-small-cell lung carcinoma by skewing macrophage populations back to M1 subsets (39). Moraes et al. demonstrated that AnnexinA1 promotes breast cancer growth and migration through regulating macrophage polarization in the tumour microenvironment (40). Furthermore, TAMs are considered as potential therapeutic targets for anti-cancer therapy $(41,42)$. A recent study also confirms that regulating macrophage polarization by effectively target to TAMs is a promising anti-cancer strategy for breast cancer (43). In line with these findings, our results showed that the expression of the M1 marker (iNOS ${ }^{+}$) was remarkably increased in MSJZD-treated mice compared with that in the control mice. However, there was no significant difference in M2 marker expression between the two groups. Therefore, we speculated that MSJZD may alter the phenotype of TAMs by upregulating M1 macrophages. Considering the key roles of TAMs in tumor metastasis, our results suggest that the anti-tumor effect of MSJZD may be demonstrated through elevating the expression of M1 markers. However, we did not further explore the detail mechanism of MSJZD in regulating macrophage polarization, and more experiments are required to understand this in future studies.

\section{Conclusions}

Our findings revealed that MSJZD promoted macrophage polarization into the M1 phenotype to stimulate anti-tumor effects, thus inhibiting tumor growth and metastasis in breast cancer. Our findings may provide new insights that contribute to therapeutic strategies against breast cancer.

\section{Abbreviations}

MSJZD: modified Sijunzi decoction

TAMs: Tumor-associated macrophages

TCM: Traditional Chinese medicine 


\section{Declarations}

\section{Ethics approval and consent to participate}

All procedures in this study were approved and supervised by the animal research ethical committee of Zhejiang Chinese Medical University, and strictly obeyed the rules of animal experiment ethic to reduced number as well as suffering of animals.

\section{Consent for publication}

All authors have read and agreed to the published version of the manuscript.

\section{Availability of data and materials}

Please contact corresponding authors for data requests.

\section{Competing interests}

The authors declare no conflict of interest.

\section{Funding}

This work was supported by the Natural Science Foundation of Zhejiang Province (Project of Youth Foundation) (No. LQ15H290004), National Natural Science Foundation of China (Grant No:81973805), Zhejiang Provincial TCM Science and Technology Project (Grant No: 2015ZA088), Zhejiang Provincial Project for the key discipline of traditional Chinese Medicine (Yong GUO, no, 2017-XK-A09, http://www.zjwjw.gov.cn/ )

\section{Contributions}

$H Z$, YG and $Z L$ designed the study, $H Z, B X$ and $X Z$ performed experiments, conceived the study, draft and revised the manuscript. $B X, X Z$ analyzed and interpreted the data. $H Z, Y G$ and $Z L$ provided the financial support. All authors read and approved the final manuscript.

\section{Acknowledgement}

Not applicable.

\section{References}

1. DeSantis CE, Fedewa SA, Goding Sauer A, Kramer JL, Smith RA, Jemal A. Breast cancer statistics, 2015: Convergence of incidence rates between black and white women. Cancer J Clin. 2016;66(1):31-42.

2. Sidaway P. Breast cancer: LAG3 expression indicates favourable outcomes. Nature Reviews Clinical Oncology. 2017. 
3. Bussard KM, Marini FC. Tumor-associated osteoblasts are major mediators in facilitating bone metastatic breast cancer cell quiescence. AACR; 2016.

4. Solinas G, Germano G, Mantovani A, Allavena P. Tumor-associated macrophages (TAM) as major players of the cancer-related inflammation. J Leukoc Biol. 2009;86(5):1065-73.

5. Mantovani A, Sica A. Macrophages, innate immunity and cancer: balance, tolerance, and diversity. Curr Opin Immunol. 2010;22(2):231-7.

6. Mantovani A, Sozzani S, Locati M, Allavena P, Sica A. Macrophage polarization: tumor-associated macrophages as a paradigm for polarized M2 mononuclear phagocytes. Trends Immunol. 2002;23(11):549-55.

7. Liu B, Wang X, Chen T-Z, Li G-L, Tan C-C, Chen Y, et al. Polarization of M1 tumor associated macrophage promoted by the activation of TLR3 signal pathway. Asian Pacific journal of tropical medicine. 2016;9(5):484-8.

8. Ruffell B, Affara NI, Coussens LM. Differential macrophage programming in the tumor microenvironment. Trends Immunol. 2012;33(3):119-26.

9. Murray PJ, Wynn TA. Protective and pathogenic functions of macrophage subsets. Nature reviews immunology. 2011;11(11):723.

10. Rolny C, Mazzone M, Tugues S, Laoui D, Johansson I, Coulon C, et al. HRG inhibits tumor growth and metastasis by inducing macrophage polarization and vessel normalization through downregulation of PIGF. Cancer cell. 2011;19(1):31-44.

11. Lewis CE, Pollard JW. Distinct role of macrophages in different tumor microenvironments. Cancer research. 2006;66(2):605-12.

12. Tai SK, Chang H-C, Lan K-L, Lee C-T, Yang C-Y, Chen N-J, et al. Decoy Receptor 3 Enhances Tumor Progression via Induction of Tumor-Associated Macrophages. Journal of Immunology.188(5):246471.

13. Sica A, Larghi P, Mancino A, Rubino L, Porta C, Totaro MG, et al. Macrophage polarization in tumour progression. Seminars in Cancer Biology.18(5):349-55.

14. Ye L, Jia Y, Ji K, Sanders AJ, Xue K, Ji J, et al. Traditional Chinese medicine in the prevention and treatment of cancer and cancer metastasis. Oncology letters. 2015;10(3):1240-50.

15. Nie J, Zhao C, Deng L, Chen J, Yu B, Wu X, et al. Efficacy of traditional Chinese medicine in treating cancer. Biomedical reports. 2016;4(1):3-14.

16. Liu J, Wang S, Zhang Y, Fan, Ht. Lin Hs. Traditional Chinese medicine and cancer: History, present situation, and development. Thoracic cancer. 2015;6(5):561-9.

17. Qi F, Li A, Inagaki Y, Gao J, Li J, Kokudo N, et al. Chinese herbal medicines as adjuvant treatment during chemoor radio-therapy for cancer. Bioscience trends. 2010;4(6).

18. Qi F, Zhao L, Zhou A, Zhang B, Li A, Wang Z, et al. The advantages of using traditional Chinese medicine as an adjunctive therapy in the whole course of cancer treatment instead of only terminal stage of cancer. Bioscience trends. 2015;9(1):16-34. 
19. Lin H, Liu J, Zhang Y. Developments in cancer prevention and treatment using traditional Chinese medicine. Frontiers of medicine. 2011;5(2):127-33.

20. X Z, JY N JG, Y Y, GL Z, MW Y, et al. Chinese Herbal Medicine as Adjunctive Therapy to Chemotherapy for Breast Cancer: A Systematic Review and Meta-Analysis.\%A Sun X. Evidence-based complementary and alternative medicine: eCAM. 2016;2016(undefined):3281968.

21. L L, Y L, J W, Q W. Chinese Herbal Medicine as an Adjunctive Therapy for Breast Cancer: A Systematic Review and Meta-Analysis.\%A Zhu L. Evidence-based complementary and alternative medicine: eCAM. 2016;2016(undefined):9469276.

22. Gao B, Wang R, Peng Y, Li X. Effects of a homogeneous polysaccharide from Sijunzi decoction on human intestinal microbes and short chain fatty acids in vitro. J Ethnopharmacol. 2018;224:465-73.

23. ZS J JR. Z. LncRNA SNHG5 enhances astrocytes and microglia viability via upregulating KLF4 in spinal cord injury. Int J Biol Macromol. 2018;120(null):66-72.

24. Liu L, Han L, Wong DY, Yue PY, Ha W, Hu Y, et al. Effects of Si-Jun-Zi decoction polysaccharides on cell migration and gene expression in wounded rat intestinal epithelial cells. British journal of nutrition. 2005;93(1):21-9.

25. Yu X, Cui Z, Zhou Z, Shan T, Li D, Cui N. Si-jun-zi decoction treatment promotes the restoration of intestinal function after obstruction by regulating intestinal homeostasis. Evidence-Based Complementray and Alternative Medicine,2014,(2014-4-28). 2014;2014(11):928579.

26. Guo J, Zhang N-S. The Method of Strengthening the Spleen and Replenishing Qi on Improving Clinical Symptoms of Patients with Advanced Ovarian Carcinoma Curative Effect Observation [J]. Journal of Practical Traditional Chinese Internal Medicine. 2012;5:031.

27. Huang X, Yu X, Chen J. Effects of Sijunzitang on Cellular Immune Functions of Colorectal Cancerous Persons with Neoadjuvant Chemotherapy. Journal of Snake. 2010;22:23-5.

28. Liang C, Zhang S, Cai Z. Effects of early intestinal application of sijunzi decoction on immune function in post-operational patients of gastrointestinal tumor. Zhongguo Zhong xi yi jie he za zhi Zhongguo Zhongxiyi jiehe zazhi = Chinese. journal of integrated traditional Western medicine. 2005;25(12):1070-3.

29. LIN Y-f WANG, C-I WANGY, WANG C-h WANG, L-x XI, Y-q, et al. Effect of supplementary Sijunzi decoction on prevention of N-methyl-N'-nitro-N-nitrosoguanidine induced gastric cancer in rat model [J]. Chinese Journal of Integrated Traditional Western Medicine on Digestion. 2006;5:003.

30. Qian J, Xie H, Guo C, Sun R, Su L, Jia J, et al. Sijunzi decoction demolition parties inhibit proliferation and induce apoptosis of human gastric cancer BGC-823 side population. Afr J Tradit Complement Altern Med. 2015;12(6):77-89.

31. Xiao H, Yang J. [Immune enhancing effect of modified sijunzi decoction on patients with colorectal cancer undergoing chemotherapy]. Chinese Journal of Integrated Traditional Western Medicine. 2011;31(2):164-7.

32. Yamaguchi H, Wyckoff J, Condeelis J. Cell migration in tumors. Curr Opin Cell Biol. 2005;17(5):55964. 
33. Hagemann T, Robinson SC, Schulz M, Trümper L, Balkwill FR, Binder C. Enhanced invasiveness of breast cancer cell lines upon co-cultivation with macrophages is due to TNF-a dependent upregulation of matrix metalloproteases. Carcinogenesis. 2004;25(8):1543-9.

34. Goswami S, Sahai E, Wyckoff JB, Cammer M, Cox D, Pixley FJ, et al. Macrophages promote the invasion of breast carcinoma cells via a colony-stimulating factor-1/epidermal growth factor paracrine loop. Cancer research. 2005;65(12):5278-83.

35. Monteiro L, Rodrigues M, Gomes D, Salgado B, Cassali G. Tumour-associated macrophages: Relation with progression and invasiveness, and assessment of M1/M2 macrophages in canine mammary tumours. Vet J. 2018;234:119-25.

36. Klingen $T A$, Chen $Y$, Aas $H$, Wik E, Akslen LA. Tumor-associated macrophages are strongly related to vascular invasion, non-luminal subtypes, and interval breast cancer. Human pathology. 2017;69:7280.

37. Yang J, Zhang Z, Chen C, Liu Y, Si Q, Chuang T, et al. MicroRNA-19a-3p inhibits breast cancer progression and metastasis by inducing macrophage polarization through downregulated expression of Fra-1 proto-oncogene. Oncogene. 2014;33(23):3014.

38. Zhang M, He Y, Sun X, Li Q, Wang W, Zhao A, et al. A high M1/M2 ratio of tumor-associated macrophages is associated with extended survival in ovarian cancer patients. Journal of ovarian research. 2014;7(1):19.

39. Kang H, Zhang J, Wang B, Liu M, Zhao J, Yang M, et al. Puerarin inhibits M2 polarization and metastasis of tumor-associated macrophages from NSCLC xenograft model via inactivating MEK/ERK 1/2 pathway. Int J Oncol. 2017;50(2):545.

40. Moraes LA, Kar S, Foo SL, Gu T, Toh YQ, Ampomah PB, et al. Annexin-A1 enhances breast cancer growth and migration by promoting alternative macrophage polarization in the tumour microenvironment. Scientific reports. 2017;7(1):17925.

41. Komohara Y, Fujiwara Y, Ohnishi K, Takeya M. Tumor-associated macrophages: potential therapeutic targets for anti-cancer therapy. Adv Drug Deliv Rev. 2016;99:180-5.

42. Pathria P, Louis TL, Varner JA. Targeting tumor-associated macrophages in cancer. Trends in Immunology. 2019.

43. Tariq M, Zhang J, Liang G, Ding L, He Q, Yang B. Macrophage Polarization: Anti-Cancer Strategies to Target Tumor-Associated Macrophage in Breast Cancer. Journal of cellular biochemistry. 2017;118(9):2484-501.

\section{Figures}




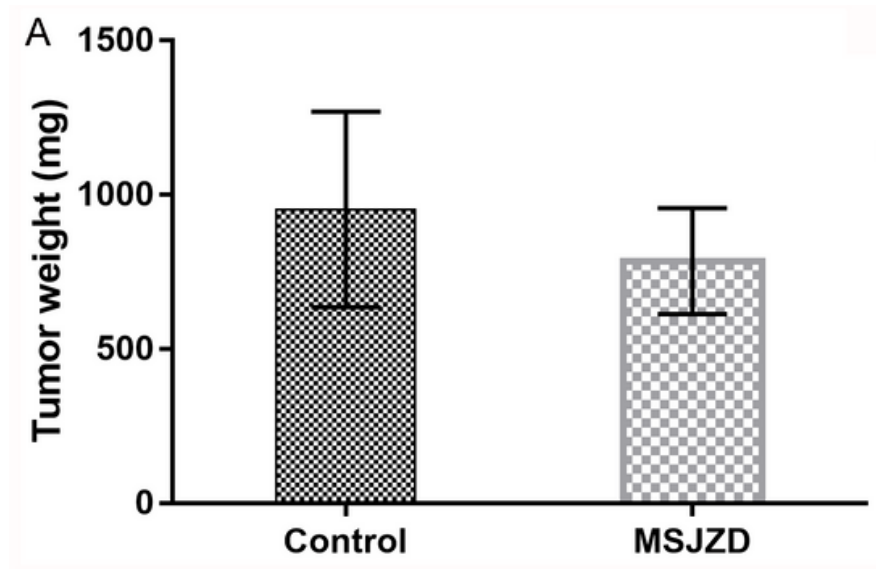

Control

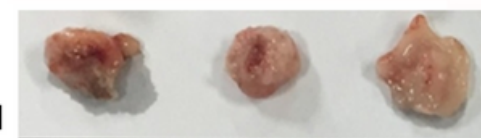

MSJZD

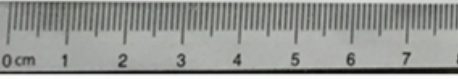

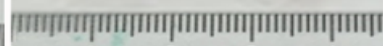
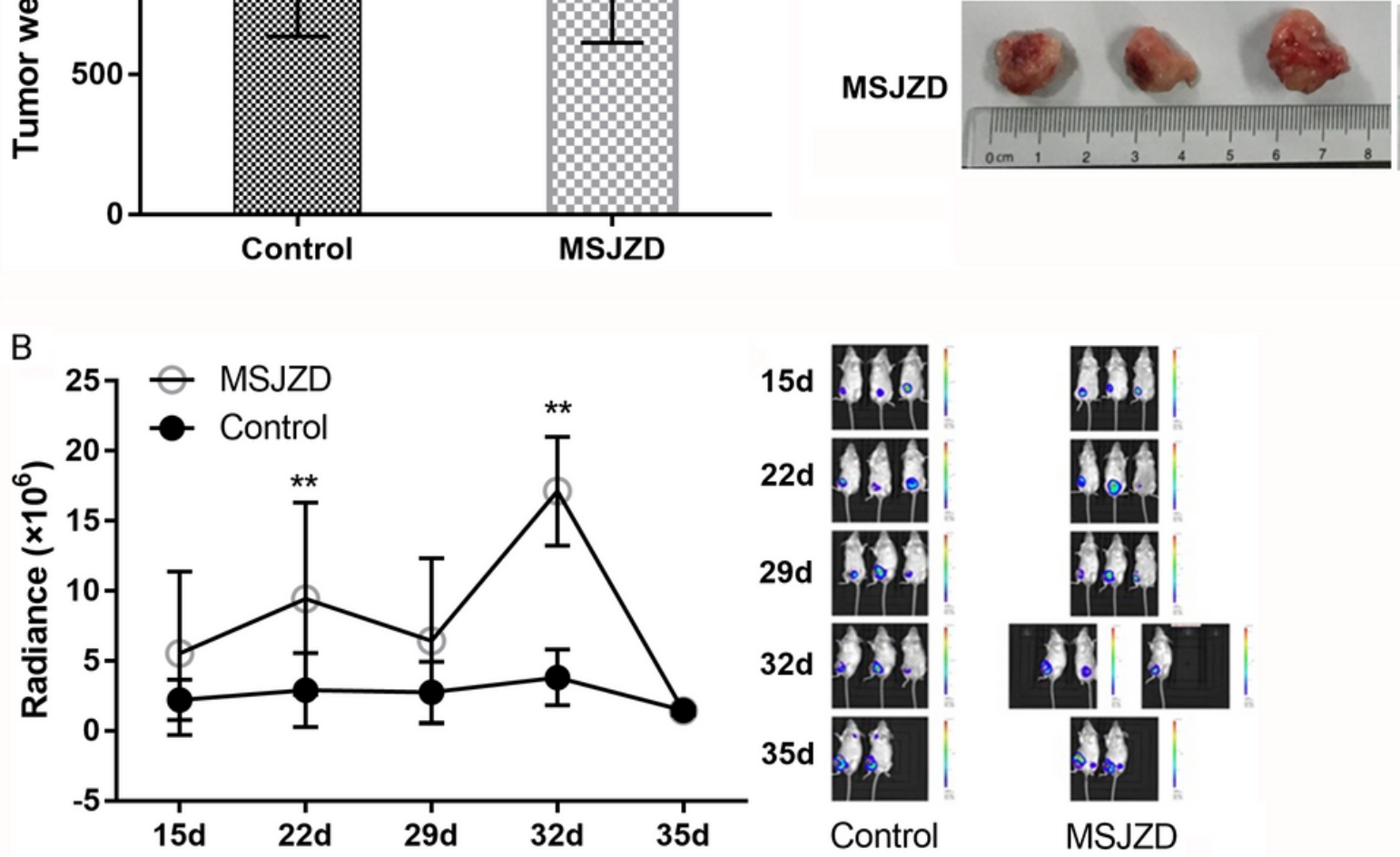

Figure 1

Effects of MSJZD on tumor growth and metastasis in the Balb/c mouse model of breast cancer. (A) The mice were sacrificed by cervical vertebra dislocation on day 35. The weights of the tumors in the MSJZD and control groups were obtained on day 35. (B) In vivo imaging study showed the radiance of fluorescence emitted from the mice $6 \mathrm{~h}$ after administration of fluorescent DiD oil in the MSJZD-treated and control mice on days 15, 22, 29, 32, and 35. MSJZD: Sijunzi decoction. ** $\mathrm{P}<0.01$ compared to control group. 

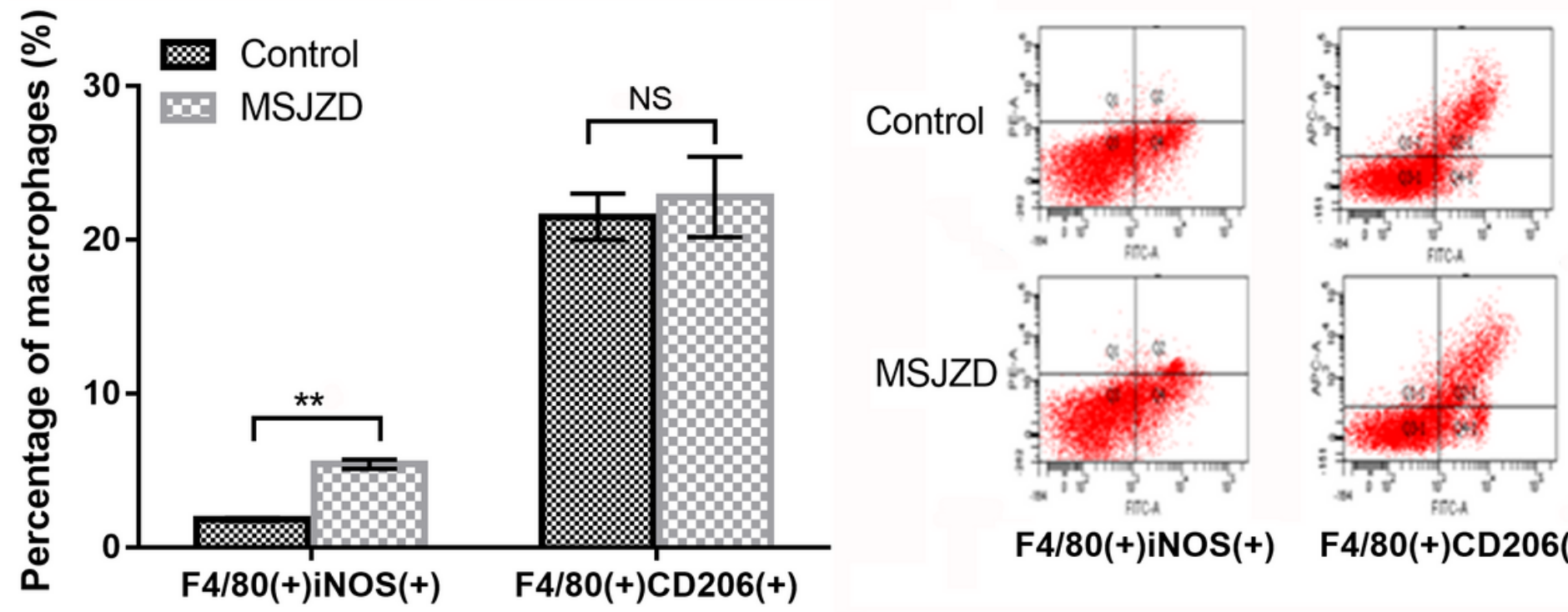

F4/80(+)iNOS(+)

F4/80(+)CD206(+)

Figure 2

MSJZD promoted macrophage polarization into the M1 phenotype. On day 35 , the mice were sacrificed by cervical vertebra dislocation. The tumor cells from the MSJZD-treated and control mice were stained with the macrophage marker F4/80, the M1 marker iNOS, and the M2 marker CD206. The polarization of $\mathrm{M} 1 / \mathrm{M} 2$ was analyzed using flow cytometry. ${ }^{*} \mathrm{P}<0.01$ compared to the control group.

\section{Supplementary Files}

This is a list of supplementary files associated with this preprint. Click to download.

- graphicalabstract.tif 